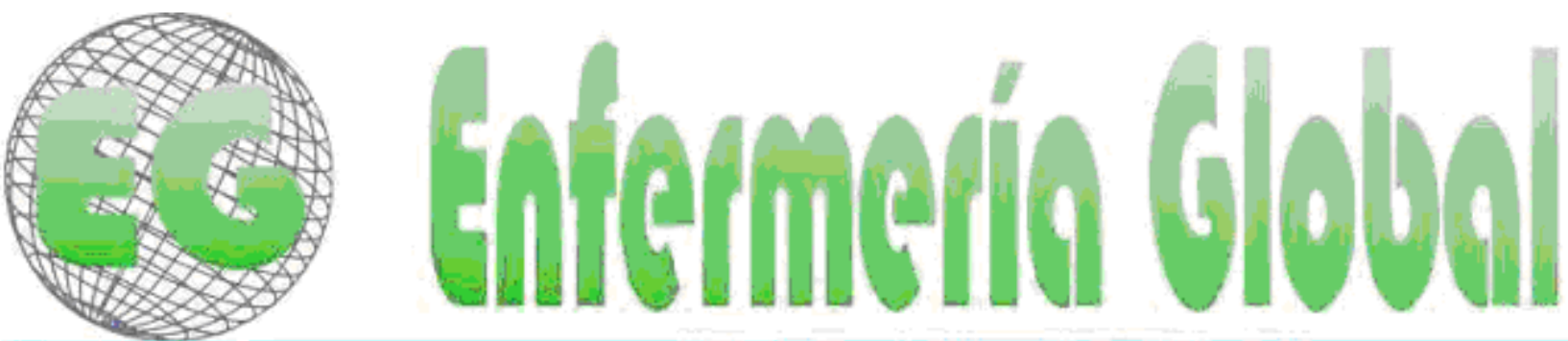

$N^{\circ} 18$

Riovista electrônica cuatrimestral de Enfermeria

Febrero 2010

www.um.es/eglobal/

\title{
CLÍNICA
}

\section{LOS FACTORES AMBIENTALES Y SU RELACIÓN CON EL BAJO PESO AL NACER EN EL EXTREMO SUR DE BRASIL}

OS FATORES AMBIENTAIS E SUA RELAÇÃO COM O BAIXO PESO AO NASCER NO EXTREMO SUL DO BRASIL

\begin{abstract}
*Stein Backes, MT., "**Mendonza Sassi, R., ***Flores Soares, MC.
*Mestre em Enfermagem / Hospital-Escola - Universidade Federal de Pelotas (UFPel). **Doutor em Epidemiologia / Departamento de Medicina Interna e Programa de Pós-Graduação em Enfermagem da Fundação Universidade Federal do Rio Grande (FURG). ${ }^{* * *}$ Doutora em Fisiologia da Reprodução / Departamento de Ciências Fisiológicas e Programa de Pós-Graduação em Enfermagem da FURG. Brasil.
\end{abstract}

Palavras-chave: poluição ambiental; baixo peso ao nascer; epidemiologia.

Palabras clave: polución ambiental; bajo peso al nacer; epidemiología.

\section{RESUMEN}

Esta investigación de casos y controles, realizada en el municipio de Rio Grande/RS, tuvo como objetivo evaluar el bajo peso al nacer (BPN) en recién-nacidos de madres que viven en las proximidades del área industrial de esta ciudad, consideradas más expuestas a la polución ambiental. Participaron de la encuesta 547 madres que alumbraron en las maternidades en los meses de abril a noviembre de 2003. La muestra estuvo compuesta de 138 casos y 409 controles. Los resultados muestran que el lugar de la vivienda materna en el área expuesta presentó razones de odds mayores para BPN tanto en el análisis bivariado (RO 1,87; IC95\% 0,95-3,66), cuanto en el análisis multivariado $(\mathrm{RO}=4,67 ; \mathrm{IC} 95 \%$ 0,95-22,90), pero los valores de $\mathrm{p}$ ubicándose mucho cerca del punto de corte establecido ( $p=0,067$ e $p=0,057$, respectivamente). De esta forma, se ve que hay una relación entre la exposición a contaminantes y BPN, pero son necesarios otros estudios para poder responder a esta cuestión con más claridad. Esos resultados muestran la necesidad de que la influencia ambiental sea considerada por parte de todo el grupo de salud, y por el enfermero en especial, tanto en lo que se refiere al planeamiento, desarrollo y acompañamiento pre-natal de las gestantes, como también, en el seguimiento de la asistencia a sus niños después del nacimiento

\section{RESUMO}

Este estudo de casos e controles, realizado no município de Rio Grande/RS, teve como objetivo avaliar o baixo peso ao nascer (BPN) em recém-nascidos de mães residentes nas proximidades da área industrial deste município, consideradas mais expostas à poluição ambiental. Foram entrevistadas 547 mães que deram à luz nas maternidades do município, durante os meses de abril a novembro de 2003. A amostra foi composta por 138 casos e 409 controles. Os resultados mostram 
que o local da residência materna na área exposta apresentou razões de odds maiores para BPN tanto na análise bivariada (RO 1,87; IC95\% 0,95-3,66), quanto na análise multivariada $(\mathrm{RO}=4,67$; IC95\% 0,95-22,90), mas os valores de $p$ situaram-se muito próximos ao ponto de corte estabelecido ( $p=0,067$ e $p=0,057$, respectivamente). Desta maneira, esboça-se uma relação entre exposição a poluentes e BPN, mas são necessários outros estudos para poder responder a esta questão com maior clareza. Esses resultados mostram a necessidade de que a influência ambiental seja considerada por parte de toda a equipe de saúde, e pelo enfermeiro em especial, tanto no que se refere ao planejamento, desenvolvimento e acompanhamento pré-natal das gestantes, como também, no seguimento da assistência de suas crianças, após o nascimento.

\section{ABSTRACT}

The study of cases and check-ups performed in Rio Grande county/RS, aimed to evaluate Low Birth Weight (LBW) in newborns of mothers residing near the industrial section in the town, and thought to be exposed to environmental pollution. 547 mothers who gave birth in hospital maternity units were interviewed between April and November 2003. The sample was made up of 138 cases and 409 check-ups. The results show that the location of the mothers residence in the area in question showed a greater odds ratio to LBW both in the bivaried analysis (RO 1.87; IC95\% 0.95-3.66) and the multivaried analysis ( $\mathrm{RO}=4.67$; IC95\% 0.95-22.90), but the $\mathrm{p}$ values were closer to the established cut off point ( $p=0.067$ and $p=0.057$, respectively). Thus a relation between exposure to pollutants and LBW is shown, although additional studies are needed to clarify this issue area. These results show that it is important for health care workers, and especially by nurses, to take into account environmental issues regarding planning, development and prenatal care of pregnant women, as well as extended assistance to their children after birth.

\section{1 - INTRODUCCIÓN}

El descubrimiento de que los factores ambientales representarían riesgos reproductivos ocurrió en los inicios del siglo XX, con relatos en Europa y en Japón, asociando el aumento del número de recién nacidos muertos, abortos espontáneos y muerte neonatal, a la exposición ocupacional de mujeres al plomo y a que los agentes ambientales pueden causar daños irreversibles en el embrión en desarrollo ${ }^{(1)}$.

Recientemente, estudios han apuntado que los factores ambientales pueden agravar la salud, interferir en las condiciones gestacionales y, consecuentemente, en la reproducción. Asociación positiva fue encontrada entre abortos espontáneos y la ocupación y local de trabajo de las mujeres y de sus maridos en una comunidad industrializada finlandesa ${ }^{(2)}$. En São Paulo, también fue constatado que los fetos sufren los efectos de la contaminación siendo encontrada asociación positiva entre la contaminación atmosférica y abortos y natimortalidad $^{(3)}$.

Las condiciones de salud del recién nacido, entre ellas el peso al nacer, son determinadas por diversos factores, complejos e interrelacionados, que se originan de condiciones biológicas, sociales y ambientales a las que la madre está expuesta durante a gestación ${ }^{(4)}$.

Algunas investigaciones ya han demostrado una asociación entre contaminación ambiental potencial y BPN. Estudio realizado en Londres, para evaluar todos los nacidos de partos únicos registrados en 1991, muestra que la contaminación atmosférica puede afectar los resultados gestacionales, desencadenado BPN y prematuridad cuando la madre está expuesta durante la gravidez, principalmente en el primer trimestre de la gestación ${ }^{(5)}$. De forma semejante, estudio poblacional realizado en seis ciudades de los Estados Unidos, evaluó la relación entre el BPN a término y los niveles de diferentes contaminantes en el aire, entre los nacidos en los años de 1994 a 1996. Los autores sugieren que niveles 
aumentados de contaminación atmosférica con $\mathrm{CO}$ y $\mathrm{SO}_{2}$ presentes en el ambiente pueden ser asociados con un riesgo aumentado para el BPN ${ }^{(6)}$.

En Seúl, en Corea, fue estudiada la relación entre el BPN y los niveles de exposición y la contaminación atmosférica, durante diferentes fases gestacionales. Los riesgos para el BPN tienden a aumentar con la exposición al CO entre el segundo y el quinto mes de gestación, con la exposición al material particulado inferior a 10 micrón en el segundo y en el cuarto mes de gestación y en la exposición al $\mathrm{SO}_{2}$ y dióxido de nitrógeno $\left(\mathrm{NO}_{2}\right)$ entre el tercero y quinto mes de gestación ${ }^{(7)}$.

En Brasil, el primer estudio realizado en este sentido ocurrió en São Paulo a través de una investigación que incluyó 179.000 nacimientos ocurridos en el año 1997. Este estudio muestra que las madres que estuvieron expuestas a mayores niveles de contaminación del aire en el primer trimestre de la gestación, tuvieron recién nacidos con peso inferior al de las demás gestantes de la capital paulista. Los responsables de la investigación acreditan que el BPN está asociado, entre otros factores, a baja oxigenación sanguínea, provocada por los contaminantes $^{(8)}$.

Otro estudio del que se tiene conocimiento, también realizado en Brasil, fue realizado en Triúnfo/RS, el cual apuntó una correlación positiva para BPN y la residencia materna próxima al Polo Petroquímico. No obstante, esa asociación no fue significativa cuando otras variables fueron incluidas en el análisis de regresión logística condicional, como humo, enfermedades crónicas y edad materna ${ }^{(9)}$.

Río Grande, municipio localizado al sur del Estado de Río Grande del Sur y que presenta más de $90 \%$ de su población habitando la zona urbana, es considerado polo nacional de fertilizantes y posee una refinería de petróleo en las proximidades del centro de la ciudad. A pesar de eso, no existen estudios que investiguen la influencia ambiental sobre la reproducción y las condiciones de nacimiento en el municipio.

Por tanto, el presente estudio tuvo como objetivo evaluar el bajo peso al nacer en RNs de madres residentes en las proximidades del área industrial de este municipio, consideradas más expuestas a la contaminación ambiental. En este artículo será dado mayor énfasis a los factores ambientales, aunque este estudio haya llevado en consideración los diversos factores de riesgo relacionados con el BPN.

\section{2 - METODOLOGIA}

Este estudio de casos y controles fue realizado en el municipio de Río Grande/RS, en el período de abril a noviembre de 2003.

Los casos fueron definidos como los recién nacidos de partos únicos, vivos o muertos, hijos de madres residentes en el municipio de Río Grande, que tuvieron su parto en las maternidades de los dos hospitales generales de la ciudad, durante el período del estudio, con BPN, o sea, pesando menos de 2.500 gramos. Fueron considerados controles los tres niños, en las mismas condiciones mencionadas, que nacieron inmediatamente después del caso, en el mismo hospital, con peso al nacer igual o superior a 2.500 gramos. En este estudio fueron incluidos los recién nacidos pretérminos a partir de 20 semanas de gestación, con peso igual o superior a 500 gramos. 
Los cálculos del tamaño de la muestra fueron realizados basándose en un estudio de casos y controles. El tamaño de la muestra fue definido para alcanzar un nivel de confianza de 95\%, un poder de $80 \%$ y un riesgo relativo de 2,50 , estando la prevalencia de exposición entre los controles en $9 \%$. Se estimó que, trabajando con una proporción de tres controles para cada caso, e incluyendo 10\% para pérdidas y 15\% para control de las variables de confusión, serian suficientes 139 casos (recién nacidos con BPN) y 417 controles (recién nacidos con peso en normal), siendo entrevistadas 547 madres.

Los datos referentes al recién nacido, así como los referentes a la madre (nombre, peso en el día del parto, entre otros), fueron levantados a partir de los registros tomados en la maternidad de cada hospital. Además de eso, un cuestionario fue aplicado a las madres todavía durante la internación hospitalaria en la maternidad, por un entrevistador entrenado. Con este cuestionario fueron investigados los factores de riesgo que podrían interferir en el resultado estudiado. También fue realizada la medición de la altura de la madre en el final da aplicación del cuestionario.

Teniendo en cuenta el costo elevado y la dificultad de la utilización de biomarcadores para una evaluación cuantitativa de la exposición, en este estudio fueron consideradas expuestas las madres que residían en las proximidades del área industrial del municipio de Río Grande, por más de nueve meses, teniendo en cuenta que el tiempo de gestación abarca nueve meses. Con el fin de delimitar la región del estudio, fueron incluidos en esta área, los Barrios Hogar Gaúcho, Navegantes, parte del Barrio Centro, parte del Barrio Getúlio Vargas (BGV), Santa Teresa, Villa Javier, Villa San Antonio y además, la Villa de la Manguera, que queda en el camino entre la refinería de petróleo y las industrias de fertilizantes. Debido a la gran extensión del BGV, fue considerada área expuesta solamente la mitad del barrio más próximo al parque industrial, comprendida entre la calle Marciano Espíndola (calle 6) y la calle Ingeniero Hetor Amaro Barcelos. Para definir esta área como expuesta, fueron también tenidos en consideración los registros del mapa de la dirección de los vientos predominantes en Río Grande, en los diversos períodos del año.

De acuerdo con los criterios antes mencionados las variables relacionadas a los factores ambientales evaluados en esta investigación fueron el local de residencia materna, el tiempo de residencia de la madre en el área considerada expuesta, o local de residencia anterior de la madre y el lugar de trabajo de la madre durante la gestación.

Los datos fueron evaluados por el método de regresión logística no condicional, obteniéndose las razones de Odds e intervalos de confianza en todos los análisis, utilizándose un nivel de significancia de 0,05. En el análisis bivariado, el efecto de cada una de las variables fue analizado aisladamente. En la regresión logística multivariada fue efectuado el ajuste para todas las variables que permanecieron en el modelo jerárquico previamente establecido, o sea, que presentaron un nivel de significancia menor que 0,20, de forma a considerar la posibilidad de confusión negativa.

\section{3 - RESULTADOS}

\section{1 - Análisis bivariado}

La Tabla 1 muestra las variables relacionadas con los factores ambientales entre casos y controles y el efecto bruto de esas variables sobre el BPN. 
El lugar de residencia presentó una RO aumentada más con un $p$ limítrofe $(p=0,067)$. Entre tanto, el lugar de residencia se asoció significativamente con la presencia de otros fumadores en casa (fuera el compañero) y el peso materno pregestacional. Entre las madres expuestas, la proporción de otros fumadores en casa fue cerca de dos veces mayor (42,5\%) en relación a las no expuestas (20,3\%). En cuanto al peso materno el pregestacional, en el área expuesta hubo una mayor proporción de madres con peso pregestacional inferior a 55 quilos $(53,8 \%)$ que en el área no expuesta (35,6\%). El lugar de residencia no estuvo asociado con la renta familiar. Ocho por ciento de las madres con mejor renta estuvieron expuestas, contra $4,7 \%$ de las madres más pobres.

Tabla 1 - Distribución entre casos y controles y efecto bruto sobre el bajo peso al nacer de las variables sobre los factores ambientales

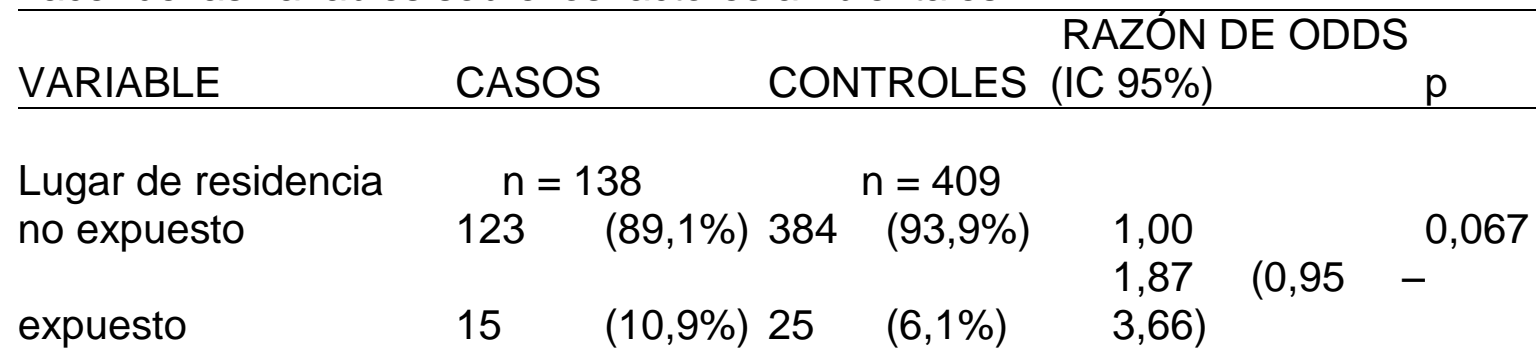

Tiempo de residencia

\begin{tabular}{|c|c|c|c|c|c|c|c|}
\hline en el área expuesta & $\mathrm{n}=$ & & & $=409$ & & & \\
\hline no expuesto & 123 & $(89,1 \%)$ & 384 & $(93,9 \%)$ & 1,00 & $(\cap \wedge 6$ & $0,058^{* *}$ \\
\hline expuesto ates 5 años & 4 & $(2,9 \%)$ & 8 & $(2,0 \%)$ & $5,27)$ & & \\
\hline expuesto más de & 5 & & & & 2,02 & $(0,92$ & - \\
\hline años & 11 & $(8,0 \%)$ & 17 & $(4,2 \%)$ & $4,42)$ & & \\
\hline
\end{tabular}

Lugar de residencia

\begin{tabular}{|c|c|c|c|c|c|c|c|}
\hline \multirow{3}{*}{$\begin{array}{l}\text { anterior } \\
\text { no expuesto }\end{array}$} & \multicolumn{2}{|c|}{$\mathrm{n}=138$} & \multicolumn{2}{|r|}{$n=407$} & \multirow[b]{2}{*}{1,00} & \multirow[b]{3}{*}{$(0,56$} & \multirow{3}{*}{$\begin{array}{r}0,8 \\
-\quad\end{array}$} \\
\hline & 123 & $(89,1 \%)$ & 365 & $(89,7 \%)$ & & & \\
\hline & 15 & $(10,9 \%)$ & 42 & $(10,3 \%)$ & $\begin{array}{l}1,05 \\
1,97)\end{array}$ & & \\
\hline -ugar de trabajo & & $n=138$ & & $n=409$ & & & \\
\hline 10 expuesto & 124 & $(89,9 \%)$ & 374 & $(91,4 \%)$ & $\begin{array}{l}1,00 \\
1,20\end{array}$ & $(0,62$ & $\begin{array}{r}0,6 \\
-\quad\end{array}$ \\
\hline xpuesto & 14 & $(10,1 \%)$ & 35 & $(8,6 \%)$ & $2,31)$ & & \\
\hline
\end{tabular}

${ }^{*}$ El tiempo de residencia en el área expuesta inferior en nueve meses fue considerado como no expuesto en este estudio

${ }^{* *}$ Valor de $p$ de la prueba de la tendencia lineal, utilizado para las variables con tres o más categorías

La media del tiempo de residencia en el área expuesta de los casos y controles fue semejante, o sea, 16 años entre los casos y 15,4 años entre los controles. El tiempo de residencia en el área de exposición también presentó valor $p$ limítrofe $(p=0,058)$ en una prueba de tendencia lineal. Cuando el tiempo de residencia en el área expuesta fue superior a cinco años, el número de casos expuestos fue cerca de dos veces mayor $(8,0 \%)$ que de los controles (4,2\%). Hubo un aumento del riesgo cuando el tiempo de exposición fue hasta cinco años $(R O=1,56)$ y, ese riesgo aumentó todavía más cuando el mismo fue mayor de 
cinco años $(\mathrm{RO}=2,02)$. La renta familiar no estuvo asociada con el tiempo de residencia en el área expuesta.

Con el fin de explorar otras consecuencias del tiempo de residencia sobre la salud de las gestantes, se realizaron análisis complementarios, no observándose asociación con ninguna de las patologías investigadas (hipertensión arterial, diabetes, anemia, infección urinaria y amenaza de aborto). Tampoco estuvieron asociados con el lugar de residencia la escolaridad materna, el hábito de fumar materno, el consumo de bebida alcohólica por la madre durante la gestación y el cigarrillo pasivo, con excepción del cigarrillo pasivo de otras personas en casa (sin considerar el compañero). Cerca de $42 \%$ de las madres expuestas, independientes del tiempo de exposición, tuvieron a alguien fumando en su domicilio, contra $20,3 \%$ de las madres no expuestas, como ya fue citado anteriormente.

El lugar de residencia anterior no se asoció con el BPN. La relación expuesto/no expuesto fue muy semejante, $10,9 \%$ de los casos eran expuestos y, 10,3\% de los controles. El lugar de residencia anterior tampoco estuvo asociado con la renta familiar. En tanto, las madres más pobres estuvieron cerca de 1,6 veces más expuestas en su lugar de residencia anterior $(14,3 \%)$ que las madres con mayor renta familiar $(9,1 \%)$.

En cuanto a la exposición en el lugar de trabajo, no fue encontrada asociación con el BPN, siendo que la proporción de expuestos en el lugar de trabajo fue semejante: 10,1\% de los casos y $8,6 \%$ de los controles. La exposición en el lugar de trabajo no estuvo asociada con la renta, aunque, las madres más pobres igualmente estuvieron cerca de 1,6 veces más expuestas $(11,6 \%)$ que las madres con hasta tres o más salarios mínimos mensuales $(7,4 \%)$.

\section{2 - Análisis multivariado}

En esta fase del análisis las variables referentes a los factores ambientales fueron ajustadas para las variables que permanecieron el modelo con $<0,20$, conforme lo explicado en la metodología, siendo ellas: presencia del compañero, escolaridad paterna, edad materna, altura e índice de masa corporal materna, intervalo interpartos, mortinatos previos y BPN previos. En ese modelo de ajuste, las variables sobre los factores ambientales que entraron el modelo de análisis fueron apenas el lugar de residencia y el lugar de trabajo materno (Tabla 2).

El lugar de residencia presentó una RO mayor a 4, pero con un valor de $p$ limítrofe al punto de corte establecido $(p=0,057)$. Debe ser destacado el aumento del efecto después el ajuste comparando con el valor bruto.

Con respecto al lugar de trabajo, hubo una reducción del efecto entre los expuestos $(R O=0,23)$, pero la asociación no fue significativa $(p=0,08)$. 
Tabla 2 - Distribución entre casos y controles del efecto bruto de las variables sobre los factores ambientales asociados con el BPN, ajustados para las variables socioeconómicas y demográficas maternas, características biológicas y factores reproductivos significativos

\begin{tabular}{llll}
\hline \multirow{2}{*}{ VARIÁBLE } & CASOS & RAZÓN & DE \\
& & ODDS & ONTROLES \\
& & AJUSTADA & (IC \\
& & $95 \%)$ & \\
\hline
\end{tabular}

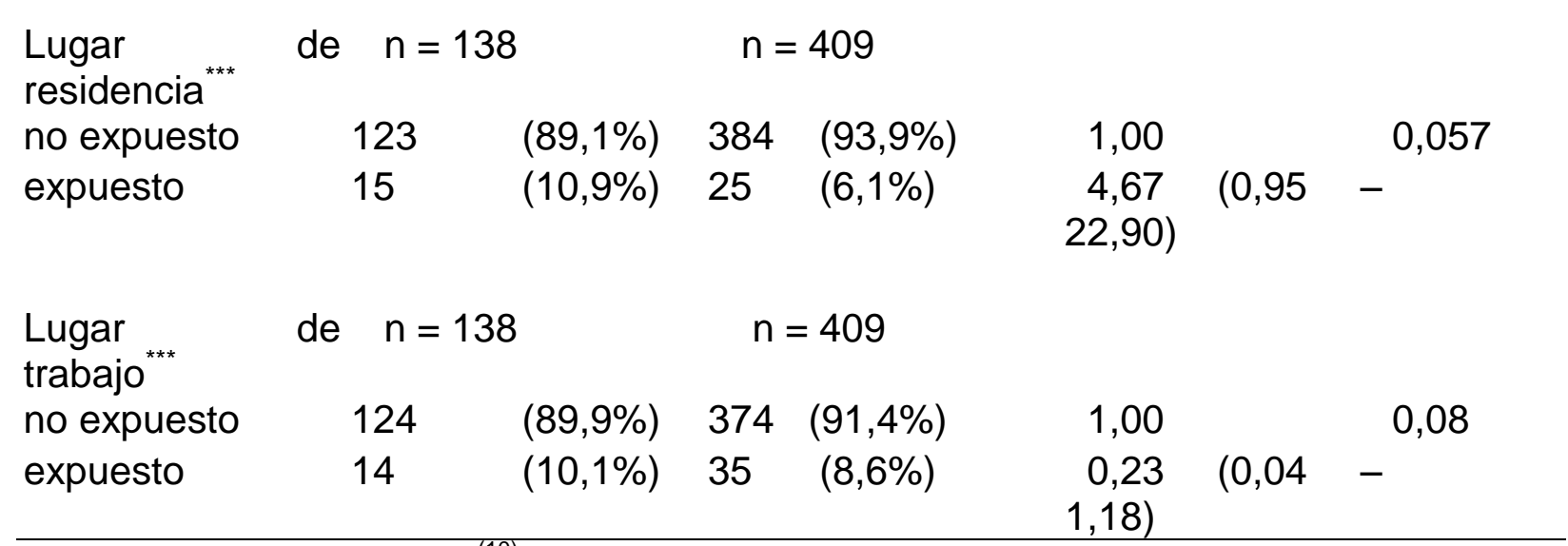

FUENTE: BACKES, $2004^{(10)}$

*** Variables que permanecieron en el modelo de análisis, ajustados para presencia del compañero, escolaridad paterna, edad materna, altura y IMC de la madre, intervalo interpartos, historia de mortinatos previos y BPN previos.

Al investigarse la presencia de malformaciones entre los RNs en este período, fue posible verificar que, en esta muestra, las mismas no estuvieron asociadas con la exposición a la contaminación ambiental. Entre tanto, las malformaciones se asociaron significativamente con el BPN. Entre los casos, 4,3\% (06) eran RNs con malformaciones, frentre apenas 0,5\% (02) de los controles. De los siete tipos de malformaciones encontrados (síndrome de Down, polidactilia en mano derecha e izquierda, malformación de los miembros con rotación externa del miembro inferior izquierdo, hidrocefalia, labio leporino y paladar figurado a la izquierda, espina bífida lumbar y meningocele y, anencefalia), hubo apenas un caso de área expuesta (polidactilia en manos derecha e izquierda).

\section{4 - DISCUSIÓN}

El lugar de residencia materna en el área considerada expuesta presentó un aumento de posibilidades para el resultado estudiado, pero el valor de $p$ fue limítrofe $(p=0,067)$. El aumento del efecto después el ajuste indica la presencia de algún factor de confusión negativa.

Uno de los factores que puede haber contribuido para la ausencia de una asociación significativa es el tamaño de la muestra, toda vez que la prevalencia de exposición entre los controles fue inferior a la estimativa inicial. Otro factor limitante puede haber sido la clasificación como expuesta y no expuesta. Es bien conocida la dificultad de medir las exposiciones ambientales ya que por su propia característica sería necesario prácticamente un monitoreo individual, lo que desde el punto de vista logístico se hace muy difícil. En este estudio se optó por medir la exposición ambiental por la localización geográfica de los participantes, lo que eventualmente puede haber producido una clasificación incorrecta no diferencial. 
Nuestros resultados indican la posibilidad de existir alguna asociación entre exposición a contaminantes ambientales en el local de residencia y nacimientos con bajo peso, aún después del ajuste con otros factores conocidos para esta resultado neonatal, pues las $\mathrm{RO}$ encontradas son altas, si bien presentan un intervalo de confianza bastante largo, indicando justamente que el $n$ no fue suficiente.

Por tanto, la localización de la residencia materna en las proximidades del parque industrial del municipio de Río Grande/RS puede ser uno de los determinantes para el nacimiento de niños con bajo peso. Estudio transversal, realizado también en este municipio, en que fue evaluado el desarrollo neuropsicomotor de 87 niños entre 0 y 12 meses de edad, en esta misma área del parque industrial, y de 83 niños de un área considerada no expuesta (o menos expuesta), en función del mapa de dirección preferencial de los vientos de esa localidad, se constató una prevalencia cerca de cuatro veces mayor de bajo peso al nacimiento entre los niños del área expuesta ${ }^{(11)}$.

Sin duda, otros estudios son necesarios para evaluar y esclarecer mejor ese efecto sobre el BPN en esta área. Se hace necesaria la realización de estudios que utilicen marcadores, además del lugar de residencia, para que puedan delimitar con mayor precisión cuales son las áreas más afectadas ${ }^{(12)}$. Por otro lado, es necesario investigar la exposición de las gestantes en los diferentes trimestres de la gestación y hasta en diferentes estaciones del año, incluyendo muestras mayores en relación al área expuesta. Los resultados hacen reflexionar también sobre el hecho de que los efectos de los contaminantes ambientales, al menos en este estudio, podrían estar siendo potencializados por el tabaquismo pasivo de otros fumadores en el domicilio durante la gestación.

El tiempo de residencia de la madre en el área expuesta también presentó valor $p$ limítrofe $(p=0,058)$. Ya en el análisis ajustado, esta variable no fue significativa. Los análisis complementarios mostraron que la historia de mortinatos previos tiende a asociarse significativamente con el tiempo de exposición. Entre tanto, hubo un número muy reducido de madres con mortinatos previos, apenas tres, las cuales residían en el área expuesta por más de cinco años. De las madres que vivían en el área de exposición por menos de cinco años, ninguna tuvo mortinatos previos. Esta asociación podría tal vez estar actuando como una variable de confusión con relación al tiempo de exposición.

El lugar de trabajo no presentó asociación con el BPN en ninguno de los análisis efectuados y el número de madres expuestas en el ambiente de trabajo también fue pequeño, apenas $10,1 \%$ de los casos y $8,6 \%$ de los controles. Al trabajar en el área expuesta, la madre no sufre los mismos efectos de la exposición que cuando reside en el área expuesta y, las mujeres, generalmente ejercen actividades menos peligrosas, comparadas con los hombres. En el presente estudio, apenas $29,4 \%$ de las madres de la muestra ejercieron algún tipo de trabajo remunerado. Por tanto, el número de madres que trabajaban en el área expuesta era pequeño. Además de eso, esas madres ejercían actividades de enseñanza, trabajo administrativo de nivel intermedio e inferior, como vendedoras y agentes de venta, auxiliares en servicios públicos y servicio domestico.

Finalmente, considerando el pequeño número de recién nacidos con malformaciones en el municipio de Río Grande/RS durante el período estudiado, se concluye que para estudiar específicamente la ocurrencia de malformaciones en este municipio, probablemente sea necesaria una muestra de mayor tamaño que la presentada en este estudio, así como un período de recolección de datos más amplio. 


\section{5 - CONSIDERACIONES FINALES}

La presente investigación epidemiológica identificó la posibilidad de la existencia de una relación entre exposición ambiental a contaminantes y BPN en el municipio do Río Grande/RS, aunque no haya sido encontrada una asociación significativa, toda vez que el valor de $\mathrm{p}$ encontrado fue un poco superior al punto de corte establecido. Se hacen necesarios en este municipio otros estudios para evaluar y esclarecer mejor el efecto de la contaminación ambiental sobre el BPN y las malformaciones, a través de la utilización de otras metodologías, como por ejemplo, el uso de biomarcadores y de marcadores locales de la contaminación, en los diferentes trimestres de la gestación y hasta incluso en diferentes estaciones del año, incluyendo muestras mayores en relación al área expuesta, principalmente en el caso de estudios que incluyen malformaciones.

En el área de enfermería el asunto ambiental ha sido abordado con otros enfoques ${ }^{(13)}$. Entre tanto, los profesionales del área de la salud están despertando la consciencia de que la contaminación ambiental generada por los procesos industriales interfiere en el proceso gestacional de las madres expuestas, ocasionado BPN, provocando malformaciones y hasta causando la muerte de embriones y fetos.

Considerando el hecho de que la residencia materna próxima al área industrial interfiere en el desarrollo gestacional de las madres expuestas, comprometiendo el peso al nacer de sus hijos, se hace necesaria una asistencia más cualificada por parte de todo el equipe de salud, significando al enfermero en especial, una atención diferenciada y más comprometida, donde la influencia ambiental también sea tenida en consideración, tanto en lo que se refiere a la planeación, desarrollo y acompañamiento prenatal de estas gestantes, como también en el seguimiento de la asistencia a estos niños, después del nacimiento. Así como en otras áreas, la práctica del enfermero volcada para la salud materno infantil debe ser en norteada por referencias teóricas, filosóficas y metodológicas, haciéndolo un profesional crítico y reflexivo sobre su ser y hacer ${ }^{(14)}$.

\section{6 - REFERENCIAS}

1 Zierler S, Theodore M, Cohen A, Rothman KJ. Chemical quality of maternal drinking water and congenital heart disease. International Journal of Epidemiology 1988; 17:589-94.

2 Hemmink K, Kyyronën P, Niemi ML, Koskinen K, Sallmén M, Vainio H. Spontaneous abortions in a industrialized community in Finland. American Journal of Public Health 1983; 73:32-7.

3 Pereira LA, Loomis D, Conceição GM, Braga AL, Arcas RM, Kishi HS, et al. Association between air pollution and intrauterine mortality in São Paulo, Brazil. Environmental Health Perspectives 1998; 106:325-29.

4 Kramer MS. Determinants of low birth weight: methodological assessment and metaanalysis. Bulletin of the World Health Organization 1987; 65(5):663-737.

5 Bobak M. Outdoor air pollution, low birth weight and prematurity. Environmental Health Perspectives 2000; 108:173-76. 
6 Maisonet M, Bush TJ, Correa A, Jaakkola JJ. Relation between ambient air pollution and low birth weight in Northeastern United States. Environmental Health Perspectives 2001; 109:351-56.

7 Lee BE, Ha EH, Park HS, Kim YJ, Hong YC, Kim H, et al. Exposure to air pollution during different gestational phases contributes to risks of low birth weight. Human Reprod 2003; 18:638-43.

8 Gouveia N, Bremner SA, Novaes HM. Association between ambient air pollution and birth weight in São Paulo, Brazil. Journal of Epidemiology; Comunity Health 2004; 58:11-7.

9 Oliveira LM, Stein N, Sanseverino MT, Vargas VM, Fachel JM, Schüler L. Reproductive outcomes in an area adjacent to a petrochemical plant in southern Brazil. Revista de Saúde Pública 2002; 36:81-7.

10 Backes MTS. O baixo peso ao nascer em recém-nascidos de mães residentes nas comunidades próximas ao parque industrial do município de Rio Grande/RS: um estudo de casos e controles. [dissertação]. Rio Grande (RS): Fundação Universidade Federal do Rio Grande (FURG) - Programa de Pós-Graduação em Enfermagem; 2004.

11 Fernandes CL. Avaliação do desenvolvimento neuropsicomotor em crianças entre $0 \mathrm{e}$ 12 meses de idade residentes nas comunidades próximas ao parque industrial do Município do Rio Grande, RS [dissertação]. Rio Grande (RS): Fundação Universidade Federal do Rio Grande (FURG) - Programa de Pós-Graduação em Enfermagem; 2004.

12 Huang YL, Batterman S. Selection and evaluation of air pollution exposure indicators based on geographic areas. Science Total Environmental 2000; 15:253:127-44.

13 Ribeiro MCS, Bertolozzi MR. A questão ambiental como objeto de atuação da vigilância sanitária: uma análise da inserção das enfermeiras nesse campo. Rev Latino-am. enfermagem 2004 outubro; 12(5): 736-744.

14 Rolim KM, Pagliuca LMF, Cardoso MVLML. Análise da teoria humanística e a relação interpessoal do enfermeiro no cuidado ao recém-nascido. Rev Latino-am. enfermagem 2005 junho; 13(.3):432-440.

ISSN 1695-6141 